\title{
ANTHROPOS
}

106.2011: 397-409

\section{Pouvoir et société}

\section{Repenser le lien social dans le Laos urbain}

\author{
Léo Mariani
}

\begin{abstract}
In Laos, the 1975 communist revolution brought an end to the constitutional monarchy. The new rulers, aware of their lack of legitimacy, quickly realized that they would have to fill the former elite's symbolic and ritual frame. This paper shows that this return to traditional forms of power conceals the main anthropological implications of the shift caused by the communist revolution, which actually altered radically and permanently the Lao mode of being in society. [Laos, power, society, gift, social change]
\end{abstract}

Léo Mariani, ethnologue, chercheur associé à l'Unité de recherches "Migrations et Sociétés" (URMIS). Après quatre ans passés au Laos, il a soutenu une thèse de doctorat intitulée "Lien social et place de l'individu dans la société lao contemporaine" et a reçu le soutien du Center for Khmer Studies (Phnom Penh, Cambodge) pour initier une recherche post-doctorale sur les Franco-Cambodgiens de deuxième génération qui s'installent au Cambodge. Il vit actuellement à Phnom Penh où il poursuit ce travail. - Publications récentes : "Du risque et de la perception de l'intentionnalité dans le don. Une proposition de typologie des rapports sociaux lao en milieu urbain" (2009) ; "Affirmation et distinction. Un exemple de mise en perspective d'une relation d'entretien" (2008) ; "Référence de valeur et valeur de référence : Le kièt et le pièp, 'pivots' des relations interindividuelles lao" (2008). Voir aussi les références citées.

\section{Introduction, cadrage théorique}

Comme l'histoire contemporaine du Vietnam et du Cambodge, celle du Laos est marquée par une rupture politique brutale qui, au cœur des années de guerre froide (1975), fit passer le pays de la tutelle d'une monarchie bouddhique constitutionnelle à celle d'un régime communiste qui, dans un premier temps, bouleversa un ordre symbolique bien établi et affaiblit une économie déjà peu développée. La prise de pouvoir du "peuple", soutenue par l'URSS et assez largement pilotée en sous-main par le voisin vietnamien, provoqua l'exode d'une bonne partie de la noblesse et de la bourgeoisie locale (vers la France, l'ancienne puissance coloniale, en particulier). Selon le Haut Commissariat des Nations Unies aux Réfugiés, parmi les 320000 Laotiens qui quittèrent le pays au cours des premières années du nouveau régime, $90 \%$ faisaient ainsi partie de la classe moyenne éduquée (Stuart-Fox $2001: 262$ ).

Après quelques années d'un dogmatisme rigide qui se frotta à une résistance passive mais persistante de la population et à un manque criant de moyens tant idéologiques que matériels, le gouvernement communiste démantela progressivement la grande majorité des réformes les plus dures et les plus symboliques qu'il avait dans un premier temps instaurées. Plus tard, à partir de la chute de l'URSS, puis de façon encore plus manifeste à la fin des années 1990, il ouvrit résolument le pays à la circulation des capitaux et des personnes, provoquant un essor économique et urbanistique sans précédent dans la région de Vientiane notamment. Le pragmatisme dont firent preuve les nouveaux gouvernants à chacune de ces étapes charnières leur permit, probablement à dessein, de consacrer une grande partie de leur attention à la conservation et à la perpétuation d'un pouvoir qu'ils s'étaient approprié au mépris de l'hérédité, parangon idéologique qui jusque-là avait présidé à la légitimation de l'autorité.

Comme le soulignent les principaux spécialistes de l'histoire contemporaine du pays (Evans 1998 ; Stuart-Fox 1996, 2005, 2006), cette volonté s'est 
traduite au niveau institutionnel par un réinvestissement progressif de la trame symbolique et rituelle à laquelle la monarchie s'était adossée et qui lui avait permis de se reproduire. Dès le début des années 80, on put ainsi voir les plus haut cadres du Parti reprendre la place de leurs prédécesseurs dans diverses cérémonies traditionnellement liées à la représentation du pouvoir. Les grandes fêtes du calendrier bouddhique par exemple, qui avaient dans un premier temps fait l'objet d'interdictions ou de larges remaniements, redevinrent ainsi un lieu privilégié de légitimation pour la nouvelle hiérarchie politique.

Considérer les choses du seul point de vue de l'institution étatique serait toutefois réducteur, car c'est dans les routines et les événements du quotidien, comme les fêtes de mariage, que le retournement de comportement des notables du nouveau régime fut peut-être le plus déterminant (Mariani 2006 ; Phoumirath 2004). La réapparition de normes et de codes anciens de représentation du statut est ainsi caractéristique d'une tendance globale parfaitement identifiable dans la plupart des circonstances importantes de la vie sociale lao. La majorité des observateurs laotiens et étrangers que je connais partagent d'ailleurs ce diagnostic dont l'évocation est récurrente dans la plupart des conversations où la question du comportement des hommes politiques est abordée.

Pourtant, derrière les nombreuses homologies factuelles qui sont ainsi relevées et qui font largement consensus, il me semble que la principale implication anthropologique de la rupture provoquée par la révolution communiste reste entièrement inexplorée. Dans cet article, je voudrais en effet montrer que cette rupture a en fait radicalement et définitivement modifié l'essence même du mode lao d'être en société en provoquant un renouvellement du principe fondateur de l'agencement social, lequel reposait jusque-là sur la présence d'un pouvoir héréditaire conçu comme le relais et le médiateur d'une volonté créatrice et légitimatrice autonome. L'ordre social étant de cette façon explicité, donné et établi depuis une source extérieure à la société, il ne pouvait en principe faire l'objet ni d'appropriations ni de modifications, tous les hommes lui étant également soumis. Or, si une telle particularité a longtemps contribué à normaliser le fait politique en le contenant dans un ordre idéologique religieux, elle fut inévitablement et radicalement affectée par l'apparition d'une élite spontanément constituée et, de fait, séparatrice d'avec la transcendance. Cette dissociation, brutale dans le cas du Laos, du politique et du religieux a eu pour conséquence d'induire un renversement complet du fondement de la société, faisant mécaniquement advenir le politique comme un "objet" autoproduit et donc autonome, identifiable en lui-même et pour lui-même : la politique (Gauchet 2005 : 19). Sous l'effet de cette ouverture, c'est également (et en conséquence) la perception de la société, là aussi comme entité autonome, et désormais comme moteur de l'invention collective (société civile), qui devint possible.

Ce renversement, qualifié de "libéral" par Gauchet $(2005: 23)$ parce qu'il ouvre structurellement l'accès de la production du sens et des règles à la société et aux individus, n'empêche en aucune façon la reproduction de comportements ataviques. Au contraire, il y incite d'autant plus vivement qu'aucun mode de légitimation alternatif (suffrage universel etc.) n'est disponible et que le nouvel agencement n'est, à la base, pas fondé en droit. Lorsqu'il advient, le "renversement libéral" ne modifie pas non plus à proprement parler la structure des rapports de pouvoir, mais plutôt la façon dont elle est animée : dans un cas, l'articulation du politique et du religieux revêt un caractère organique, elle constitue l'essence même de l'unité hiérarchique, dans l'autre elle est d'ordre mécanique, fondamentalement liée à la nécessité d'acquérir une légitimité qui fait initialement défaut et à laquelle tout un chacun peut désormais aspirer. Du point de vue strictement formel, le contraste entre les deux configurations est donc caractérisé par un simple décalage relatif à l'ordre dans lequel pouvoir et légitimité s'impliquent. Les répercussions potentielles d'une telle évolution sont pourtant considérables. On peut par exemple estimer qu' elle est à l'origine du développement, à différents degrés, de l'idéologie libérale définie comme "vision d'ensemble de la société organisée autour du fait libéral" (Gauchet 2005 : 24) ${ }^{1}$ dans les sociétés occidentales (Gauchet 2005 ; Dumont 1978). En permettant la reconnaissance du pouvoir des hommes sur l'organisation de leur société, et donc l'existence de lois soustraites à la conscience immédiate (car n'étant pas données d'emblée), elle contribue aussi, comme nous le verrons, à rendre une investigation de type sociologique possible (Gauchet 1979).

Quoi qu'il en soit, le replacement du cas laotien dans le cadre d'analyse qui vient d'être ébauché permet de faire ressortir un paradoxe intéressant pour le propos : d'un côté le gouvernement communiste se trouve (sans le savoir) à l'origine d'une mutation (d'une libéralisation) subtile de l'agencement social, et d'un autre, il n'a d'autre choix, pour se perpétuer, que d'entraver toutes les perspectives

1 Ce qui ne préjuge pas de la possibilité du développement d'alternatives idéologiques, sur la même base. 
qu'elle permet d'ouvrir. C'est en effet un trait partagé des régimes totalitaires que de s'opposer au développement de l'individualisme en essayant toujours de rétablir une subordination des sujets à la société envisagée comme un tout (Dumont 1977). Le régime politique laotien actuel est pour cette raison le siège d'une contradiction irréductible, car le simple fait de son existence ouvre la porte à des forces qu'il ne peut en principe pas tolérer. Comme souvent, le totalitarisme "combine, sans le savoir, des valorisations opposées", s'acharnant à recréer un tout à partir d'éléments discrets : “d'où l'accent démesuré, féroce, sur la totalité sociale. D'où la violence et son culte, moins encore parce qu'il faut obtenir la soumission là où la subordination - qui demande l'accord général des citoyens sur les valeurs fondamentales - est hors d'atteinte, que parce que la violence habite les promoteurs du mouvement eux-mêmes, déchirés qu'ils sont entre deux tendances contradictoires et condamnés par là à tenter désespérément de poser la violence à la place de la valeur" (Dumont 1977 : 22). Si le niveau de totalitarisme développé par l'État laotien doit sans aucun doute être relativisé en regard de celui qui est évoqué dans ce portrait, il n'en reste pas moins que le contrôle exercé est suffisamment important pour que les rares expressions émanant de la société civile ou d'initiatives individuelles aient fait et continuent de faire l'objet de sanctions souvent exemplaires.

Les répercussions du changement opéré en 1975 au Laos restent donc insidieuses, ce qui explique peut-être qu'elles n'aient à ma connaissance jamais été relevées. Elles tiennent essentiellement au sentiment plus ou moins conscient qu'en réinvestissant un système symbolique et politique qui, jusque-là, reposait en théorie sur la présence d'une noblesse de sang aux postes de pouvoir, les nouveaux dirigeants du pays se sont vu attribuer des devoirs statutaires (qui sont pour eux des obligations) qu'ils n'ont pas la légitimité d'acquitter. S'il est en effet "normal" (sans ambiguïté), du point de vue idéologique, qu' un noble se conduise avec ostentation, puisque son comportement est alors conforme avec une norme institutionnalisée, le "parvenu" qui adopte un comportement analogue offre au contraire une prise parfaite à la suspicion car, dans son cas, l'intéressement n'est pas impliqué par un état originel, mais le fonde par définition. L'individualisation totale des choix des acteurs est, en principe, devenue possible, ce qui a pour conséquence de nourrir un doute grandissant sur les motivations des élites, de plus en plus fréquemment soupçonnées d'être intéressées et égoïstes. La nuance introduite par la rupture politique de 1975 se livre ainsi dans toute sa complexité, car le développement de la suspicion ne repose pas tant sur une réalité que sur une potentialité. Comme je l'ai déjà évoqué plus haut, les membres des nouvelles élites mettent au contraire un soin tout particulier à assumer les prescriptions symboliques liées à leur statut à travers une surenchère dont on comprend ici qu'elle n'est pas sans lien avec une volonté réelle de prouver leur sincérité. En tout état de cause, le passage charnière de 1975 n'a pour l'instant pas particulièrement modifié les comportements humains, mais a modifié en premier lieu leur perception. À Vientiane, le sentiment de défiance qui en découle paraît se diffuser à la société entière, accentuant la méfiance portée à tous les individus qui manifestent par exemple une trop grande générosité ou, plus globalement, qui donnent l'impression d'avoir un comportement non conforme avec leur statut. Il a aussi pour implication de contribuer à une plus grande contractualisation des rapports sociaux (Mariani 2009), car ceux-ci n'étant plus intégralement règlementés et par conséquent sécurisés "du dehors", ils doivent nécessairement l'être "du dedans".

Dans les lignes qui suivent, le propos va donc s'attacher à revenir sur cette présentation théorique en tentant de mieux définir ce que le renversement libéral décrit plus haut semble avoir changé dans la gestion et l'appréhension du statut social et donc dans l'inscription des individus au sein de la société lao urbaine. A cette fin, je reviendrai d'abord sur le contenu du rapport vertical qui liait le souverain bouddhiste à ses sujets (ainsi que sur le rapport de vassalité en général), puis sur les spécificités de la relation en principe horizontale entre les gens du commun. Je tenterai ensuite de montrer que la dissociation du politique et du religieux provoquée par la révolution de 1975 a contribué à faire fusionner ces deux types de liens, concrétisant une tendance que les changements économiques du milieu du XXe siècle avaient déjà suscitée, sans toutefois pouvoir lui donner une forme définitive. Pour terminer, je reviendrai sur les implications de ce passage sur la dialectique individu/société en questionnant les notions de générosité et de don.

\section{Sur l'origine symbolique de la société et les spécificités de la relation de vassalité lao}

Bien que la rareté et la discontinuité des sources historiques qui décrivent le mode traditionnel d'organisation spatiale et politique de la société lao ${ }^{2}$ doivent

\footnotetext{
2 Et plus généralement de l'ensemble des sociétés taï (Nguyen The Anh 1998), auquel appartiennent les sociétés lao comme les sociétés thaï, shan, etc.
} 
inciter à la prudence, un certain nombre d'observations semblent faire consensus. Parmi les auteurs qui se sont intéressés au sujet, tous soulignent ainsi l'importance descriptive et heuristique du concept de müang ${ }^{3}$, unité de base des systèmes politiques taï dont la principale caractéristique réside dans le fait qu'il désigne tout à la fois une ville et le territoire qui en dépend. En principe, un müang est de surcrôit lui-même composé d'un agrégat d'autres müang plus modestes qui lui sont subordonnés, cette relation pouvant être plusieurs fois reproduite selon l'importance de l'unité principale jusqu'au niveau ultime et irréductible du village (ban). Il en découle qu'un royaume et une principauté vassale, comme une capitale et une ville de province, sont désignés par le même terme malgré leur différence d'échelle. Le müang ne peut donc être défini que par son centre, la zone d'influence relative de celui-ci, étroitement dépendante de la figure du gouvernant et de sa capacité à combiner les différentes ressources du pouvoir, étant seulement sous-entendue, car par définition mouvante. Le müang principal était gouverné par un souverain de "mandat divin" auquel des seigneurs de müang secondaires (tiao müang, "princes", ou litt. "propriétaires" de müang), généralement issus des aristocraties locales, vouaient allégeance. Le lien de vassalité ainsi défini reposait sur un système plutôt classique d'engagements réciproques : les inférieurs acquittant diverses taxes et services en échange de la protection de leur supérieur et des privilèges découlant de ses liens avec la surnature.

On ne saurait, quoi qu'il en soit, comprendre et rendre compte des spécificités d'une telle organisation sans évoquer le point essentiel sur lequel elle se distingue de la relation féodale du Moyen Âge européen. Si les deux agencements sont en effet comparables au niveau des droits et devoirs qu'ils attribuent aux différents acteurs, ils divergent radicalement quant à la nature du rapport entre seigneurs et vassaux qui, dans le cas présenté ici, ne reposait pas en principe sur une base héréditaire (Hanks 1962 : 1250). La subordination de certains müang à d'autres, plus importants, était essentiellement le fruit d'alliances qui n'avaient a priori rien de définitif car elles pouvaient être rompues. La puissance du centre était donc directement corrélée à l'intensité de son attractivité, laquelle tenait plus à la stature symbolique du souverain et à l'entrelacs complexe de liens clientélistes qu'il avait su tisser qu'à tout autre déterminant. Qu'elle vienne à décroître (ou qu'une autre plus importante se présente)

3 Le terme müang est aujourd'hui utilisé pour caractériser les districts. et le subordonné, seigneur (Stuart-Fox 2005 : 5) ou simple villageois (Taillard 1992), était libre de changer de tutelle.

Du point de vue de l'organisation du pouvoir et des significations qui peuvent lui être associées, la possibilité ainsi donnée aux subordonnés de privilégier un rapport plutôt qu'un autre en fonction des capacités et du rayonnement du dominant est déterminante, car en liant intimement la dépendance à un choix, la contrainte au libre arbitre, elle permet d'ouvrir le rapport à une forme de concurrence qui explique l'instabilité souvent relevée des systèmes politiques taï. Un tel schéma relationnel ne peut en tout état de cause être compris dans les limites d'une typologie binaire qui opposerait, comme le propose Testart (1997), deux formes de dépendances : une dépendance de fait, établie de façon conditionnelle car relevant d'un choix personnel (un consommateur doit se rendre chez le boucher s'il désire manger de la viande), et une dépendance d'état, inconditionnelle, illustrée par le Moyen Âge français où le serf dépend d'un seigneur sans autre motif que sa seule condition et ne peut, "seul, de par lui-même, ni de par quoique ce soit qui vienne de lui, sortir de cet état. Ce qui ne signifie pas, bien sûr, que cet état ne soit pas modifiable, ni que le dépendant ne puisse en sortir, mais il ne peut en sortir que pour des raisons qui lui sont extérieures ... Le vassal ne peut se libérer du lien qui l'unit à son seigneur de son propre chef, mais il l'est automatiquement du fait de certaines fautes commises par ce seigneur" (Testart 1997 : 52). Cette typologie, qui est posée en termes exclusifs, négatifs d'une part (le serf est libéré par les fautes de son seigneur ou de son souverain) et positifs de l'autre (le consommateur est dépendant de son boucher - et de la viande qu'il lui vend - seulement s'il le souhaite), est, comme on peut le constater, dépourvue de toute considération dialectique. Elle peut d'ailleurs paraître symptomatique d'une opposition analytique sur laquelle les sciences sociales ont souvent buté, attachées à appréhender les faits sociaux par les prismes antagonistes de la vision holiste (les individus sont soumis à des normes sur lesquelles ils n'ont aucune influence) et d'une vision reposant sur le paradigme de l'individualisme méthodologique (les individus sont libres de leurs choix, motivés par leur seul intérêt). Or, si une partie des raisons qui permettent au subordonné de s'extraire de la relation sont bien, ici, et comme le mentionne Testart, "extérieures" à lui (le seigneur est moins attractif), le principe d'attractivité a précisément pour corollaire de rendre envisageable une sortie du rapport en positif, car l'attractivité, même si elle se définit selon des critères objectifs, n'est pas une constante mais une 
donnée relationnelle. Elle est dépendante de l'offre en quelque sorte. Dans ces circonstances, l'individu est libre de choisir un rapport qui, en revanche, ne l'est pas du point de vue systémique ; libre de déterminer lui-même les conditions d'une dépendance de fait. Cet Homo hierarchicus, tel que le qualifiait Dumont, jouit d'une certaine autonomie de choix au sein d'un univers de possibles relationnels par essence polarisé. ${ }^{4}$ Le rapport de vassalité lao est de ce fait simultanément rapport d'état et rapport conditionnel, il ne peut être réduit ni à l'un ni à l'autre et relève donc, pour privilégier une belle formule de Caillé (2004 : 153), d'une forme d' "inconditionnalité conditionnelle".

Comme cela a déjà été avancé, tout l'équilibre de l'organisation qui vient d'être présentée reposait fondamentalement sur la présence d'un souverain médiateur, personnalisation d'un ordre cosmique et religieux légitime découlant de l'enchevêtrement de trois sources idéologiques distinctes :

- D'abord, le souverain était l'héritier de l'ancêtre mythique Khoun Borôm, envoyé sur terre par son père Phya Thêng (roi du ciel) pour y remettre de l'ordre et qui, devenu roi, confia le royaume du Laos (Müang Lan Sang) aux soins de l'aîné de ses sept enfants (Pavie 1898).

- L'autorité royale s'appuyait par ailleurs sur la relation privilégiée que le monarque entretenait avec l'esprit tutélaire du müang (phi müang), à qui appartenait la terre. Le roi en gérait le culte et le phi le reconnaissait comme gouvernant et médiateur. De la même façon, l'intégralité de la hiérarchie politique et administrative était doublée par une hiérarchie de phi qui contribuait à l'inscription de l'État dans le territoire.

- Le dernier fondement idéologique sur lequel reposait la légitimité du souverain provenait du bouddhisme, au sein duquel il représentait la plus haute instance vivante, incarnation de la loi du karma selon laquelle statut social et vertus morales sont directement corrélés.

Bien qu'en instituant un ordre social indépendant de toute volonté humaine, ces différentes sources idéologiques contribuaient à inscrire l'inégalité au principe même de l'être-ensemble, elles concour-

4 Si l'on conserve la métaphore choisie par Testart, on pourrait formuler les choses de la façon suivante : s'il est vrai qu'un consommateur se rend chez son boucher s'il désire de la viande, il est en revanche obligé de manger, il n'a pas le choix. A cette fin, les formes de sa dépendance sont multiples car elles peuvent être rattachées à différents types d'accès à la nourriture (épicerie, marché, activité de prédation, agriculture, etc.) raient aussi très directement à moraliser et contraindre l'exercice du pouvoir en normalisant et en ritualisant explicitement la fonction royale. Les règles appliquées au souverain étaient par ailleurs déclinées dans de nombreux "codes", en usage dans les müang, et qui réglementaient très précisément les rapports entre inférieurs et supérieurs, entre maris et femmes, entre villages et müang, etc. ${ }^{5}$ De cette façon, c'est toute la signalétique du statut qui se trouvait contrainte par une obligation de consensus rendant l'occupation d'une position sociale notoire impossible autrement que par l'exaltation d'un répertoire de codes sociaux partagés. L'opposition courante entre "culture des élites" et "culture populaire" se construisait donc de façon linéaire, via l'intensité avec laquelle étaient investis les signes du statut et non par l'intermédiaire de variations qualitatives, ce qui, en simplifiant l'appropriation par tout un chacun des signes et dispositions du statut, facilitait dans une certaine mesure les itinéraires de promotion sociale.

\section{La société des hommes, les relations sociales au niveau villageois}

Représentant depuis toujours la plus petite unité administrative lao, le village (ban) constitue l'horizon principal de l'immense majorité de la population du pays. Les villes elles-mêmes n'ont connu qu'une expansion très limitée qui a contribué à la préservation des échanges avec les campagnes. De toute évidence, le Laos était et demeure un pays profondément façonné par une culture rurale que l'appartenance d'une frange grandissante de la population aux zones urbaines n'a que très partiellement mise à mal. Les relations interindividuelles concrètes trouvent donc l'essentiel de leurs racines dans ce contexte villageois qui a influencé les rapports sociaux en général.

Contrairement à ce qui s'observe dans les sociétés non étatiques des Tay non bouddhistes, où l'organisation sociale est structurée selon un découpage lignager, l'unité de base du système lao, "la seule unité familiale existante" (Condominas, 2000 : 71), était constituée par la famille conjugale et sa descendance directe. En raison de l'abondance de terres caractérisant cette zone de l'Asie du Sud-est, chaque maisonnée d'un village contrôlait la surface qu'elle était en mesure de cultiver, ses

\footnotetext{
5 Pour beaucoup, ces “codes” prétendaient découler d'une série de règles édictées par Khoun Borôm et rapportées dans un texte écrit, "Kotmay thammasat Khoun Bourôm" (Ngaosyvathn 1996), alors que d'autres, comme le "Kôtmay thammasat bouhan", étaient d'inspiration bouddhique.
} 
droits fonciers expirant à l'arrêt de l'exploitation. S'il est manifeste, comme le fait remarquer Taillard (1992 : 308), qu'une telle particularité permettait de condamner toute forme de spéculation en empêchant l'accumulation de la principale source de richesse par des individus particuliers, les relations entre familles étaient en tout état de cause, et en principe, égalitaires, fondées sur la confiance et la reconnaissance plus ou moins explicite de l'intérêt commun.

Au cœur de la vie sociale des villages lao se trouvait le monastère bouddhique, institution aux dimensions religieuses et pratiques, mais aussi pôle identitaire autour duquel se concentrait l'attention des habitants pour qui, comme le montrent Condominas (2000) et Pottier (2004), il était l'objet de toutes les planifications et projets d'investissement prioritaires. Le monastère, dans lequel la plupart des surplus économiques étaient réinvestis, constituait en fait l'élément clé de l'organisation sociale villageoise, assumant une charge intégratrice et redistributive déterminante dont la notion de boun (mérites) permet le mieux de rendre compte. Dans l'ordre cosmique bouddhique, le rang, la position, la situation, sont en effet toujours relatifs, car les êtres humains sont perpétuellement voués, par leurs actes, à en changer. Pour progresser dans l'échelle des valeurs et, au terme de plusieurs existences, parvenir à sortir du cycle des renaissances, les hommes sont incités à accumuler des mérites spirituels (litt. "faire des mérites", hèt boun) dont l'acquisition est corrélée au respect et à l'exaltation d'un registre de normes et de prescriptions au sein duquel les dons faits à la pagode et à la communauté des moines occupent une place de choix. Les bonzes se chargent ensuite de médiatiser la transformation des biens matériels en acquis spirituels puis de redistribuer, à travers une offre diversifiée de services (éducation, médecine, services religieux etc.), les subsides engrangés. C'est donc toute la société villageoise qui, par le passé, bénéficiait des dons que recevait le monastère. Comme on peut le soupçonner, la générosité du commun des mortels ne répondait pourtant qu'assez rarement aux considérations métaphysiques de la doctrine, le bénéfice de prestige immédiat que les conduites méritoires permettaient d'emmagasiner jouant, du point de vue psychologique, un rôle nettement prépondérant (Pottier 2004 : 84). La logique du boun impliquait toutefois que les antagonismes suscités par un tel enjeu contribuent paradoxalement à l'équilibre dynamique du système, car "[en] transposant du plan matériel au plan spirituel la compétition sociale, le boun établit une nouvelle logique où les biens sont accumulés au profit de la communauté toute entière.
Plus les mérites, et donc le prestige, sont recherchés, plus les richesses sont redistribuées à l'intérieur du village et plus la solidarité s'en trouve renforcée" (Taillard 1979 : 49).

Pendant longtemps, le contexte économique lao s'est caractérisé par la faiblesse des surplus dégagés au niveau villageois, lesquels dépendaient essentiellement des excédents de riz produits lors de la récolte annuelle. En général, ils étaient ensuite réinvestis dans le monastère, participant à la reproduction sociale et à l'expression de la solidarité communautaire. A quelques exceptions près, les différences économiques au niveau villageois se trouvaient de la sorte relativement nivelées, l'ascendant d'un individu particulier restant le plus souvent ponctuel et irrégulier. Implicitement, l'usage du capital économique à des fins politiques s'en trouvait régulé.

Chaque village avait son propre chef, appelé nay ban (chef de village) ou pho ban (père de village). Dans la région de Vientiane, il était en général choisi à l'unanimité par les chefs de famille réunis en assemblée (passum ban), son élection nécessitant, même a minima, l'acquiescement de chacun des participants. En toute logique, le nay ban était le plus souvent pourvu d'une personnalité respectée et consensuelle, susceptible d'incarner au mieux les qualités de médiation et de conciliation exigées par la fonction. Il ne jouissait en effet d'aucun pouvoir, son influence étant cantonnée dans les limites d'une autorité "naturelle" pour laquelle il avait du reste été élu. Le chef de village était durablement soumis à l'approbation des villageois et n'occupait ses fonctions qu'aussi longtemps que ces derniers lui accordaient leur confiance. Lorsque celle-ci venait à manquer, il prenait le plus souvent seul l'initiative de renoncer à ses prérogatives, s'épargnant ainsi la perte de face dramatique qu'une destitution aurait occasionnée.

Quoique le modèle villageois à tendance égalitaire (le terme "égalisatrice" me paraît plus approprié) dont le portrait vient d'être rapidement esquissé ait probablement toujours dû concilier avec la tentation de rendre héréditaires les différences acquises, il semble toutefois qu'il ait persisté dans une forme très proche de celle qui vient d'être décrite jusqu'aux premières décennies du XXe siècle, lorsque le pays amorça véritablement son ouverture à l'économie de marché. Dans la plaine de Vientiane, cette évolution eut pour principale conséquence d'élargir et de multiplier les accès à la production de surplus pour toute une frange de la population urbaine qui, pourvue de moyens démultipliés, entreprit de détourner à son profit les institutions traditionnelles d'expression et d'acquisition du prestige (dans un premier temps, principalement le vat). 
L'accroissement des revenus liés à l'activité économique et l'introduction de nouvelles modalités d'accès aux ressources matérielles ont de cette façon concouru à la pérennisation de différences fondées sur le capital économique et donc favorisé le développement d'une stratification sociale (Pottier 2004 : 91). La laïcisation croissante de ' État $^{6}{ }^{6}$ dont l'avènement d'un régime communiste constitue de ce point de vue l'expression ultime, n'a fait que renforcer le processus amorcé en provoquant un afflux de nouveaux notables dans tous les lieux d'accès aux moyens légitimes d'expression de la légitimité ; lieux désormais appropriés selon un mode personnalisé et donc clientéliste de redistribution.

\section{Éléments de synthèse, une question de prestige et de pouvoir}

Si l'on s'impose maintenant un premier exercice de synthèse, on remarque que le pouvoir politique lao a pendant longtemps été réservé à certaines personnalités de statut héréditaire (le roi, les nobles) alors qu'il était inaccessible au commun des mortels. Pour l'essentiel, le statut des villageois était le fruit d'une construction dont l'âge et l'opposition entre sexes constituaient les seuls éléments a priori immuables. L'expression (et l'évolution) statutaire était ici intimement associée à un consensus de points de vues qui pouvait certes faire l'objet de manipulations, mais qui, par définition, se devait d'être préservé. De cette manière, si la recherche de prestige façonnait sans l'ombre d'un doute la vie communautaire, les antagonismes qu'elle sous-tendait étaient en partie apaisés par leur irrégularité et par la nécessité d'un consentement que certaines institutions, comme le monastère bouddhique, se chargeaient par ailleurs de faciliter. Ainsi, même lorsqu'un personnage comme le chef de village était choisi pour des motifs associés à une forme de reconnaissance durable, son autorité se trouvait singulièrement contrôlée et limitée. En tout état de cause, il ne se trouvait jamais libre d'exercer une contrainte sur ses administrés. Aux personnages désignés par l'hérédité re-

6 En 1893, c'est la France, alors en pleine expansion coloniale, qui força le Siam (actuelle Thaïlande) à lui céder l'Ouest du Cambodge et tous les territoires lao situés à l'est du Mékong qui, à ce moment là, subissaient sa domination. Le traité qui en résulta fut deux fois révisé (1904 et 1907), avec la cession par le Siam de certains territoires supplémentaires, situés à l'ouest du grand fleuve. Ainsi, l'ironie du sort veut que, sans une intervention étrangère, le Laos n' existerait peut-être pas aujourd'hui. Ce sont en tous cas les Français qui instaurèrent (1947) le régime de monarchie constitutionnelle qui fut confirmé par la Conférence Internationale de Genève (1954) et perdura (avec des ajustements) jusqu'en 1975. venait donc le pouvoir politique (assorti d'un grand prestige) ; aux villageois le prestige comme horizon principal. En approfondissant l'analyse de cette répartition, on constate par ailleurs que si la première relation procède du devoir, d'une forme de mandature, la seconde repose quant à elle essentiellement sur la volonté. La distinction ainsi établie permet donc d'associer très précisément l'exercice du pouvoir politique à une forme de privilège (l'hérédité) qui ne peut, et c'est fondamental, en aucun cas être choisie.

Il apparaît ensuite que le développement économique du Laos et la laïcisation croissante de son administration ont progressivement contribué à décloisonner le prestige du pouvoir dans les zones de la géographie sociale où le second était en temps normal strictement contrôlé. Ainsi, l'introduction de moyens économiques nouveaux, liée à l'accroissement du nombre de fonctionnaires modestes, sans légitimité, a-t-elle soutenu le processus de hiérarchisation de la société villageoise en excluant des modalités traditionnelles d'expression du statut tout un pan de la population, au profit d'une bourgeoisie économique et administrative qui se mit à préempter avec une intensité croissante les médias de l'expression sociale. Ceux qui possédaient du prestige ont alors aspiré à le convertir en pouvoir et ceux qui, comme les fonctionnaires nommés sans hérédité ni légitimité, disposaient du pouvoir, ont dû acquérir du prestige. C'est donc l'automisation progressive du champ économique qui dissémina les premiers jalons d'une forme de libéralisation des rapports sociaux. Dans un second temps, la révolution communiste vint en quelque sorte affranchir définitivement le système de l'emprise du droit héréditaire (le renversement libéral) à laquelle il était encore soumis en substituant une élite spontanément constituée à la noblesse traditionnelle. Elle contribua de la sorte à parachever la fusion de la relation verticale des hommes avec les détenteurs du pouvoir et de la relation horizontale des hommes entre eux. La répartition du pouvoir et du prestige devenue obsolète, le mode clientéliste de constitution des réseaux sociaux qui, par le passé, ne concernait pour l'essentiel qu'une élite héréditaire, se trouva de fait appliqué à tous ; les aspirations des individus, auparavant contraintes de se réaliser dans les limites de l'accumulation du prestige, constituant désormais la matrice même des rapports de pouvoir et, par extension, des rapports sociaux en général. Chacun est par définition devenu producteur de son individualité, de son statut et, plus globalement, de l'image qu'il renvoie à la société.

L'objectif des paragraphes qui suivent est de montrer que cette évolution pourtant très profonde 
n'a pas eu, pour l'instant au moins, d'incidence radicale sur les comportements. Je crois en revanche qu'elle a très directement affecté la confiance diffuse et généralisée que l'existence d'une source symbolique surplombant la société humaine permettait d'apporter au sein des relations sociales.

\section{Un exemple, à propos du développement de la suspicion}

Si l'on choisit maintenant d'adopter temporairement une perspective théorique plus interactioniste que celle que j'ai privilégiée jusqu'ici, on pourrait émettre l'hypothèse que la plus évidente et la plus essentielle des conséquences de la rupture libérale qu'a connue le Laos est d'avoir ouvert, en principe et disons structurellement (mais aussi philosophiquement), la possibilité pour les membres de la société de devenir intégralement les auteurs légitimes de leur individualité comme des normes sociales. Notons qu'en insistant sur la légitimité de l'acte, cette formulation évite tout préjugé sur la capacité partout et toujours observée des humains à influer sur les règles de l'être en société et à les manipuler très concrètement, comme sur le fait qu'ils s'y emploient ou non effectivement. La question posée serait donc plutôt : dans quelle mesure ont-ils la légitimité pour le faire et, lorsque c'est le cas, d'où tirent-ils et comment obtiennent-ils cette qualité ? Il en découle une série de problèmes qui, prenant la légitimité dans le sens (large) d'une autorité à être et agir au sein d'un groupe d'une façon qui soit reconnue par lui, met la dialectique individu/société au centre du questionnement.

Dans cette optique, l'une des difficultés posées par le cas laotien relève de la collision et de l'impossible conjugaison d'une idéologie qui a tendance à associer cette légitimité à un don (l'hérédité) avec une configuration structurelle nouvelle qui, en la faisant dépendre d'un choix, la rend en principe accessible à tout un chacun. J'ai pour ma part été frappé de constater combien, pour nombre de Lao, la générosité manifestée par les membres des nouvelle élites paraissait souvent davantage problématique en soi que leur mainmise pourtant exclusive sur les domaines du pouvoir. Une telle observation me semble en tous cas déterminante dans le cadre de cette argumentation, car elle montre bien que c'est l'illégitimité de l'articulation entre le politique et le social qui est jugée et non, en premier lieu, l'autonomie désormais avérée du champ politique par rapport à la société. Pour le regard émique, l'évocation plus ou moins explicite d'élites dépourvues de la légitimité qui leur permettrait d'assurer le rôle social qui normalement échoit aux détenteurs du pouvoir passe très clairement avant la question de la gouvernance, qui accapare en revanche de façon récurrente l'attention des observateurs occidentaux. Ce point de vue a donc notamment ceci d'intéressant qu'il désigne très directement et en tout premier lieu le hiatus qui s'est fait jour sur la base du renversement libéral. De cette façon, il met en valeur l'attention toujours très présente que les Laotiens portent aux impératifs sociaux qui sont associés au statut, plus que l'intérêt qu'ils accordent à la chose politique.

Afin d'étayer empiriquement cette analyse et d'illustrer les effets du renversement libéral, je souhaite à ce niveau présenter un événement de la vie sociale lao qui, depuis quelques années, est devenu un véritable phénomène de société dans les zones urbaines : les réceptions de mariage. Ces fêtes, qui se sont surajoutées à la dramaturgie établie du mariage lao, sont apparues vers le milieu du XXe siècle. Très en vogue jusqu'en 1975, elles furent d'abord interdites par les nouveaux dirigeants avant de réapparaître vers la fin des années 80 à la faveur de l'assouplissement idéologique du régime (Mariani 2006 ; Phoumirath 2004). Elles sont ensuite progressivement devenues le lieu par excellence d'expression et de légitimation pour les personnalités de grand standing à qui elles offrent une occasion unique de démontrer leur générosité et leur puissance. Les plus grandes soirées peuvent ainsi réunir jusqu'à 2000 personnes, ${ }^{7}$ favorisant plus que tout autre événement de la vie sociale lao le déploiement intégral des réseaux sociaux des organisateurs. Pour les plus modestes et/ou les plus raisonnables, cet événement a généralement lieu au domicile de l'un des mariés (comme c'est souvent le cas dans les zones rurales), alors que les familles plus aisées louent une salle dans l'un des très nombreux établissements spécialisés de Vientiane.

L'organisation d'une réception coûte cher à ses promoteurs et conduit régulièrement à la vente de biens familiaux voire à des emprunts conséquents. À terme, l'investissement suscité par la soirée est toutefois plus ou moins amorti par la contribution financière des gens présents, car l'invitation est lancée par l'intermédiaire d'une enveloppe marquée du nom de l'invité et dans laquelle il convient de glisser une somme d'argent qui sera introduite dans une urne le soir du dîner. Il va de soi que le montant finalement attribué dépend de multiples paramètres dont les plus évidents concernent bien entendu la

7 Ce chiffre semble seulement tributaire de la capacité des lieux d'accueils. Il est par ailleurs à mettre en perspective avec la population de Vientiane qui se situe aux alentours des 300000 habitants. 
nature et la qualité du lien qui unit les protagonistes, selon qu'il est d'ordre professionnel, familial, amical, ou clientéliste (voire souvent anonyme ${ }^{8}$ ). Malgré la diversité des motivations qui sont susceptibles de résulter de ce faisceau de déterminants, il apparaît que le prix supposé du repas constitue de l'avis général le point de repère à partir duquel le choix est adapté, ainsi que la contribution minimale dictée par le code de l'honneur. Cette somme n'étant en général pas connue précisément, elle peut cependant faire l'objet d'une évaluation qui laisse en définitive beaucoup de latitude à des invités par ailleurs très sollicités. ${ }^{9}$ Comme les organisateurs les plus fortunés mettent de surcroît un point d'honneur à situer leur investissement hors de portée des capacités financières de la plupart de leurs invités, il en résulte que le total des contributions ne permet à ma connaissance jamais de couvrir les frais engagés (en moyenne, il en rembourse entre un tiers et la moitié). Une telle situation, qui est pourtant bien connue, a toutefois ceci d'intéressant qu'elle n'empêche pas le développement d'une forme de défiance à l'égard des élites, qui sont systématiquement soupçonnées d'agir selon un intérêt égoïste que l'équation logique entre le prix de revient du repas et la somme des contributions viendrait justement motiver. ${ }^{10}$ Pour l'essentiel, les accusations et le doute dont les organisateurs de grandes soirées font l'objet procèdent donc d'une sorte de fantasme que seul un mécanisme intellectuel rationaliste vient étayer, mais qui n'a aucun contenu empirique. La contradiction ainsi mise au jour est d'autant plus intéressante que les comportements et les motivations des élites contemporaines diffèrent en réalité très peu de celles de leurs prédécesseurs (ils les ont d'ailleurs copiés) qui, dans les décennies du milieu du XXe siècle, avaient eux-mêmes initié le développement des réceptions de mariage sans pour autant faire l'objet d'une telle défiance. La mise en pers-

8 Les gens cherchant souvent à avoir le plus d'invités possibles, il n'est pas rare qu'ils invitent des connaissances très lointaines, brièvement rencontrées dans le cadre du travail par exemple. Certains tentent aussi de faire venir des personnes de grand standing qu'il n'ont jamais rencontrées, mais dans les services desquels ils travaillent (un haut fonctionnaire par exemple), ou avec qui il entretiennent un lien familial parfois très ténu.

9 Pendant la saison des mariages, qui dure environ neuf mois, il est fréquent de recevoir plusieurs invitations par semaines, même pour une personne de standing modeste. Dans ces conditions, il est difficile d'honorer toutes les sollicitations, tant physiquement que financièrement. Notons cependant qu'une absence doit en général être justifiée et qu'elle n'exempte pas du versement d'une somme d'argent, qui peut toutefois être plus modeste que lorsqu' on assiste effectivement à la soirée.

10 Avec un peu de chance, s'ils possèdent des relations fortunées, ils sont susceptibles d'engranger des bénéfices. pective de ces différents éléments me semble donc corroborer l'existence d'un achoppement plus profond qui peut je crois s'expliquer par la disparition du critère de l'hérédité. Longtemps, celui-ci a en effet permis d'associer la générosité à une obligation normative (faite vertu) que les élites devaient respecter avec toute l'exemplarité que leur statut imposait. En liant de fait explicitement le contenu de cette générosité au niveau statutaire de chacun, il permettait de normaliser la défiance en la contenant dans un ordre hiérarchique idéologique. En principe, chacun était ainsi tenu d'exprimer sa générosité relativement à des capacités et des spécificités plus ou moins directement instituées par sa naissance. La disparition du critère d'hérédité a en quelque sorte dégagé le choix (et les questions qu'il soulève) de la structure sociale, laissant à chacun la possibilité de le provoquer et/ou d'y répondre, et donc de choisir contextuellement les modalités d'expression de sa générosité. À propos des réceptions de mariage, la question ne serait donc pas tant de se demander si les élites prérévolutionnaires étaient ou non intéressées (elles l'étaient probablement) que de montrer que l'institution, en tant qu'expression de la société participant à sa reproduction, ne pouvait contenir idéologiquement l'idée du remboursement.

\section{De la générosité au don, une défiance idéologique}

La question de la générosité est étroitement corrélée à celle du don. En suscitant le dévoilement de l'intentionnalité et des motivations, la préséance du choix sur la norme, le renversement libéral semble de fait avoir insinué le doute dans les rouages sociaux de l'altruisme, affectant jusqu' aux échanges les plus informels de la vie quotidienne et accroissant d'autant le nombre de Laotiens que le don embarrasse. Désormais convaincus 'qu'on ne donne jamais vraiment", que "les dons sont toujours intéressés" ou encore que "le vrai don n'existe pas", ces derniers se trouvent de plus en plus fréquemment dans l'incapacité de recevoir, inaptes à offrir leur confiance et à croire en la sincérité de la générosité que les autres manifestent. La plupart de ceux que je connais n'hésitent pas à attribuer cet état à la disparition de la spontanéité qui, selon eux, caractérisait la société lao du passé. Celle-ci aurait en somme été victime d'une forme de "désenchantement" que la modernité et le contact avec les cultures occidentales contribueraient à expliquer. Or, plutôt que de stigmatiser la tournure assez conventionnelle de ces témoignages en réduisant leur explication à l'argument d'une nostalgie générationnelle partout ob- 
servée, il me semble qu'on peut ici souligner le rôle non négligeable que joue, dans la formulation de telles inquiétudes, l'effacement de la confiance diffuse que l'extériorité de la source symbolique de la société permettait d'apporter aux relations sociales. ${ }^{11}$

Pour le point de vue anthropologique, attaché à relativiser chaque expérience empirique dans le cadre oscillatoire d'un mouvement dialectique entre la société de l'observateur et les sociétés étudiées, l'argument de la disparition du don est en tous cas intéressant à plusieurs égards. Dans un premier temps, je souhaite ici insister sur les nombreuses analogies qu'il partage avec le point de vue d'un grand philosophe français, Jacques Derrida. Pour ce dernier, on le sait, l'objectif était de sortir résolument de la dimension sociale du don en tant qu'il est créateur de dette, pour accéder à des préoccupations plus profondément éthiques et philosophiques concernant l'existence ou non d'un don véritable. Ses conclusions sont résumées dans quelques passages très souvent cités qui me semblent à bien des égards très proches des objections de mes informateurs : “pour qu'il y ait don, il faut qu'il n'y ait pas de réciprocité, de retour, d'échange, de contre-don ni de dette". En outre, "si l'autre me rend ou me doit, ou doit me rendre ce que je lui donne, il n'y aura pas eu don ... Pour qu'il y ait don, il faut que le donataire ne rende pas, n'amortisse pas, ne rembourse pas, ne s'acquitte pas, n'entre pas dans le contrat, n'ait jamais contracté de dette" (1991 : 24-26). Enfin, pour que le don soit éventuellement possible, "il faut, à la limite, qu'il [le donataire] ne reconnaisse pas le don comme don [sinon] cette simple reconnaissance suffit pour annuler le don" (1991 : 26).

Ces extraits du texte de Jacques Derrida ont déjà fait l'objet d'abondantes discussions, spécialement en France où l'analyse quelque peu hyperbolique du philosophe a été très largement critiquée par des auteurs qui en temps normal ont plutôt tendance à s'opposer (Testart 2007 ; Caillé 2007). Au passage, il n'est pas inintéressant de remarquer que l'accueil réservé aux propositions de Derrida dans les pays anglo-saxons semble en revanche avoir été résolument plus chaleureux, révélant ainsi une distribution géographique et donc socio-culturelle antagoniste qui, je crois, repose en tout premier lieu sur le niveau de crédit accordé, de part et d'autre de la Manche et de l'Atlantique, à l'individualisme comme idéologie (voir ci-dessous). Il me semble

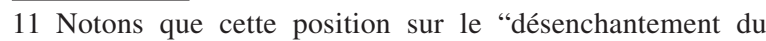
monde", que les développements de Marcel Gauchet ont jusqu'ici permis de justifier, s'inscrit de façon naturelle dans une filiation wéberienne (comp. Weber 1959). d'ailleurs que la convergence de points de vues aussi contextuellement éloignés que ceux de Jacques Derrida et de certains de mes informateurs doit elle aussi être comprise relativement à la question de l'individualisme, dans la mesure où elle signale l'existence d'une marque intellectuelle (et intellectualisante) commune que "le niveau de libéralisation" de la société dans laquelle est produit le point de vue me paraît favoriser.

D'abord, il convient en effet de souligner que les différentes appréciations dont il est question se rejoignent autour d'un même postulat implicite qui pourrait se résumer de la façon suivante : le don n'existe pas car il fait invariablement l'objet d'un contre-don, ou du moins parce qu'il suscite toujours la réalisation d'un contre-don. Le cycle du don et du contre-don repose donc sur un principe de réciprocité qui est mû par le sentiment de dette. Nonobstant l'apparente évidence, une telle supposition n'a en réalité rien d'immédiat car elle découle d'une observation et/ou d'une régularité, mais pas, a priori, d'un rapport de causalité explicite, connu de tous, comme c'est le cas dans l'échange. Fondamentalement, c'est l'observateur, le regard étique, qui effectue la conjonction entre les deux moments du cycle, et c'est d'ailleurs précisément une part importante de la contribution de Mauss aux sciences sociales que d'avoir été le premier, en Occident, à mettre aussi concrètement ce lien en évidence (puis à tenter de le conceptualiser). Il n'y a donc en principe aucune immédiateté ni aucune systématicité dans l'association du don et du contre-don, et le regard émique peut ne jamais la percevoir. Pour la discerner, il doit à son tour adopter une posture d'observateur, seule susceptible de lui permettre d'intellectualiser la structure de réciprocité sur laquelle repose le don et de lui faire au passage reproduire la découverte de Mauss. L'épanouissement d'un tel point de vue, dont on ne peut nier qu'il est proprement anthropologique, est, je crois, très largement favorisé par le renversement libéral qui, en dévoilant l'intentionnalité et en lui attribuant un statut central parmi les motifs de l'action, élargit l'accès aux lois sociologiques qui, autrement, sont dissimulées à tout un chacun.

Comme pour toute découverte d'ordre "scientifique", le problème est ensuite interprétatif, car pour Mauss, si le don relève bien, comme l'échange, d'une structure de réciprocité, il ne s'y réduit pas. C'est d'ailleurs précisément sa dimension sociale qui fait système (Caillé 2004) et qui permet ainsi de placer l'indétermination au cœur de la production et de la reproduction sociale. Pour Derrida en revanche, comme pour la plupart de mes informateurs, le phénomène sociologique semble confondu 
avec la production logique que constitue sa structure ; ce qui pourrait s'expliquer dans le premier cas par un présupposé vieux d'au moins un siècle et qui est désormais constitutif de la société d'où est émis le point de vue, et dans le second par une déduction (probablement appelée à se muer en présupposé) provoquée par la mutation de la société vers un modèle plus individualiste. Une observation qui est à l'origine fondamentalement intellectuelle, car établie sur la perception étique d'un mécanisme soustrait à toute conscience immédiate (le lien entre don et contre-don), est de cette façon instituée au principe d'un acte dont la compréhension globale ne peut pourtant ignorer le témoignage émique de ceux qui le réalisent. Or, si l'on adopte une telle posture et qu'on privilégie donc une démarche plus constructiviste, on constate que les circonstances dans lesquelles le don "n'est plus reconnu comme tel" sont en réalité nombreuses, et ce même dans les sociétés occidentales. C'est par exemple souvent le cas lorsque le don est contextualisé, normé, quand il advient comme une conséquence de la relation et non comme une amorce. Il n'est pas rare, alors, qu'il n'induise aucun sentiment de dette, voire qu'il ne soit plus désigné comme don (cf. Mariani 2009). En omettant de préciser que le don est en général dépendant d'un paramétrage social parfois très contingent qui lui sert de référent mais que les acteurs sociaux n'intellectualisent pas nécessairement, le point de vue de Derrida retranche donc la dimension normative de la réalité en la concevant comme une évidence, un donné de la conscience.

La mise en perspective ainsi réalisée avait en premier lieu pour objectif d'introduire une idée selon laquelle, en instaurant une sorte de distance critique avec la réalité sociale, certains de mes informateurs font (commencent à faire) de la philosophie ou de la sociologie sans le savoir. Les limites d'une telle appropriation se situent à mon sens dans l'usage qu'ils sont susceptibles d'en faire, car une fois le rapport virtuel entre don et contre-don établi et intelligibilisé, il devient d'autant plus compliqué à vivre qu'il est commode de le concevoir dans les termes d'une logique économiciste pour laquelle don et contredon ne constituent qu'un simple travestissement de la relation d'échange. Le don devient alors un danger permanent (dû à la dette - conscientisée - qu'il véhicule) qu'il convient soit d'éviter soit d'accepter en connaissance de cause et/ou à dessein. C'est, je crois, le choix auquel les individus laotiens sont de plus en plus fréquemment confrontés, contraints de jouer le jeu social au sein d'un cadre désormais dépourvu de garde-fous symboliques, alors que le don et la générosité étaient par le passé très souvent normalisés. Aujourd'hui, chacun est confronté quoti- diennement à la possibilité que la volonté des autres ne coïncide avec aucun cadre légitime, ce qui explique à mon avis le développement progressif d'un point de vue désocialisé, pris entre un idéal économiciste qui a tendance à rationaliser les rapports relevant du don d'une part, et un idéal philosophique qui conçoit toute forme d'altruisme dans les termes d'une pureté inaccessible d'autre part (cf. aussi Mariani 2009). Les deux ont en commun de rejeter la question des normes sociales et l'on retrouve ici, en gestation, le point de vue de l'individualisme méthodologique, qui est avant tout un point de vue d'individualités accomplies sur ce qui fait société ; et le point de vue complémentaire du philosophe post-moderne, lui aussi individualité accomplie, qui projette la pensée au-delà de toute considération sociologique. Si l'on met ces figures en perspective avec les évolutions du contexte laotien, il me semble légitime de supposer qu'elles constituent, chacune à leur manière et de façon complémentaire, les expressions scientifiques les plus abouties de l'idéologie individualiste, une idéologie parfaitement étique pour laquelle la société ne peut découler que d'une harmonisation naturelle des intérêts ou, devrait-on dire, des consciences. Une telle hypothèse pourrait au passage expliquer l'accueil plus chaleureux que les anthropologues et les sociologues des pays où cette idéologie meut les fondements même de l'organisation politique et sociale réservent aux théories qu'elle produit.

\section{Conclusion}

Pour conclure, je voudrais brièvement revenir sur un récit d'origine thaï qui, dans un contexte très proche, illustre, je crois, certaines des proposition qui ont jusqu'ici été réalisées. Il fut présenté par Jory (1996) lors d'un colloque, sans aucune mention chronologique, pour illustrer, c'était l'avis du conférencier, la fonction socio-économique du don dans la société thaïlandaise traditionnelle :

Il est question d'un homme riche qui souhaite marier son fils à une femme intelligente. À cette fin, il fait une annonce à laquelle plusieurs candidates répondent. Pour décider laquelle obtiendra le droit d'épouser son fils, il leur pose une seule et même question : quel usage feraientelles d'un gros poisson s'il leur était demandé de nourrir leur famille pendant toute une année ? Une première répond qu'elle en ferait une saumure, une seconde qu'elle le salerait et une autre qu'elle le ferait sécher. La dernière objecte qu'elle cuisinerait un grand plat qu'elle distribuerait à tout le village. L'homme, très intrigué, lui demande alors comment elle pense pouvoir nourrir toute sa famille 
pendant une année de cette façon. À quoi la femme répond qu'en donnant, on peut s'attendre à recevoir et donc que la famille serait nourrie "en retour", ce pourquoi elle est finalement choisie.

La morale de cette histoire me paraît difficile à restituer et rien ne dit en outre qu'elle soit positive. En tout état de cause, elle ne me semble pas spécifiquement révélatrice "de la fonction socio-économique du don dans la société thaïlandaise traditionnelle", mais bien plutôt d'une "fonction" universelle du don, celle de provoquer un contre-don. Partout dans le monde, inviter quelqu'un à manger, qui plus est dans un contexte d'interconnaissances, aurait probablement des implications comparables à celles qui sont ici décrites. C'est donc en tenant compte de cette observation qu'il convient de rechercher la clé du récit : en soulignant que l'argument de la candidate ne relève pas tant d'un modèle de pensée propre à une société que d'une connaissance précise liée à la compréhension d'un ressort sociologique. Pour s'en convaincre, il n'est d'ailleurs qu'à imaginer l'opprobre qu'une telle allégation aurait provoqué si les villageois en avaient pris connaissance (la question du dévoilement de l'intentionnalité et de l'intérêt).

Ceci établi, il me semble donc que c'est plus probablement la rationalité dont fait preuve la jeune femme, la conscience qu'elle possède de l'existence d'un rapport de causalité entre don et contre-don qui fait l'objet de l'attention du père du futur époux. Peut-être simplement parce que cette femme fait preuve d'intelligence, d'originalité, ou parce que, dotée d'une telle connaissance, elle serait d'autant plus susceptible de servir les intérêts de sa famille que les autres ne la possèdent pas (si un tel savoir est mis en évidence, c'est probablement qu'il n'était pas commun). On pourrait d'ailleurs ici se questionner sur le choix narratif d'un chef de famille présenté comme un "homme riche", et qui n'est décrit ni comme un seigneur ni comme un noble : l'histoire souligne-t-elle à l'origine péjorativement les calculs d'une bourgeoisie parvenue ? Il y a probablement là matière pour une investigation approfondie, mais ce récit permet de restituer certains des ressorts analysés dans cet article. Il contribue aussi à montrer que le renversement libéral ne constitue qu'un facilitateur et un accélérateur pour le développement de points de vue qui ont toujours existé.

En tout état de cause, il me semble que l'articulation entre pouvoir et société au Laos ne peut être complètement comprise sans prendre en compte un tel changement qui, s'il ne provoque pour l'instant quasiment que des effets de réaction, fera tôt ou tard l'objet d'appropriations de plus en plus nom- breuses par la société civile et les individus qui la composent.

Sans le garde-fou que constituait, en dernière instance, l'hérédité, de telles aspirations, qui ne datent pas d'aujourd'hui, contribuent à provoquer l'autonomisation de certains groupes sociaux. Si les nobles étaient en effet dépendants d'une idéologie globale et englobante qui, en dernière instance et malgré l'existence d'aspirations individualistes non réfrénées, les contraignait à jouer un rôle intégrateur, les nouvelles élites ne sont plus dépendantes que de leurs choix et de leur pouvoir de conviction. Ainsi, là où, par le passé, les grands mariages sollicitaient jusqu' au plus simple des roturiers, contribuant en quelque sorte à la reproduction de la société dans son ensemble, les nouvelles fêtes ignorent de plus en plus souvent ceux qui, dans une perspective de production des élites, ne présentent aucun intérêt. Les élites urbaines sont donc de plus en plus couramment amenées à se parler à ellesmêmes, posant de la sorte les jalons pour l'apparition de groupes sociaux constitués autour de valeurs qui ne sont pas partagées et qui, parce qu'elles réservent symboliquement (et non pas concrètement) l'accès de la représentation sociale à une certaine population, remettent en cause toute la structure de la société lao (Mariani 2006, 2008).

Reste enfin un dernier résultat que me semble suggérer l'accélération récente du développement d'un point de vue proche de celui de Jacques Derrida dans la société lao urbaine. Celle-ci contribue en effet à rappeler la dimension contextuelle, idéologique et finalement ethnocentrique des théories scientifiques. Sur le principe, ce constat permet aussi de pointer l'absurdité des débats qui reposent sur l'application exclusive de telle ou telle méthode qui, souvent, relève plus du modèle que de l'outil. L'individualisme méthodologique postule ainsi une société "d'individus méthodologiques" alors que la méthode holiste a tendance à hypostasier cette réalité en mettant l'accent sur la norme. En s'opposant au lieu de se concilier, les deux décrivent un type idéal de société auquel la réalité emprunte en permanence mais ne se réduit jamais.

\section{Références citées}

Caillé, Alain

2004 Marcel Mauss et le paradigme du don. Sociologie et sociétés 36/2: 141-176.

2007 Anthropologie du don. Le tiers paradigme. Paris: La Découverte.

Condominas, Georges, et Claude Gaudillot

2000 La plaine de Vientiane. Étude socio-économique. Paris: SevenOrients; Paul Geuthner. [1959] 


\section{Derrida, Jacques}

1991 Donner le temps. Vol. 1: La fausse monnaie. Paris: Éditions Galilée.

Dumont, Louis

1977 Homo aequalis. Génèse et épanouissement de l'idéologie économique. Paris: Gallimard.

1978 La conception moderne de l'individu. Notes sur sa genèse, en relation avec les conceptions de la politique et de 1'État, à partir du XIIIe siècle. Esprit 14: 18-54.

Evans, Grant

1998 The Politics of Ritual and Remembrance. Laos since 1975. Chiang Mai: Silkworm Books.

\section{Gauchet, Marcel}

1979 De l'avènement de l'individu à la découverte de la société. Annales. Économie, Sociétés, Civilisation 34/3: 451463.

2005 La condition politique. Paris: Gallimard.

Hanks, L. M., Jr.

1962 Merit and Power in the Thai Social Order. American Anthropologist 64: 1247-1261.

Jory, Patrick

1996 Corruption, the Virtue of Giving, and Thai Political Culture. In: Proceedings of the 6th International Conference on Thai Studies. Theme I: Globalization: Impact on and Coping Strategies in Thai Society; pp. 111-126. Chiang Mai: Chiang Mai University.

\section{Mariani, Léo}

2006 Les personnalités politiques, la politique et les mariages au Laos. La légitimité retrouvée. ASEANIE 17: 29-60.

2008 Affirmation et distinction. Un exemple de mise en perspective d'une relation d'entretien en contexte lao. ethnographiques.org 16 (septembre 2008). <http://www. ethnographiques.org $>$ [17.02.2011]

2009 Du risque et de la perception de l'intentionnalité dans le don. Une proposition de typologie des rapports sociaux lao en milieu urbain. Revue du Mauss permanente (25 septembre 2009). <http://www.journaldumauss. net/spip.php?article550> [17.02.2011]

\section{Ngaosyvathn, Mayoury}

1996 An Introduction to the Laws of Khun Borom. An Early Prototype of the Organisation of T'ai Society and Life. In A. Huxley (ed.), Thai Law, Buddhist Law. Essays on the Legal History of Thailand, Laos, and Burma; pp. 73-80. Bangkok: White Orchid Press.
Nguyên, Thê Anh

1998 La féodalité en Asie du Sud-Est. In: É. Bournazel et J.-P. Poly (eds.), Les féodalités; pp. 683-714. Paris: PUF.

Pavie, Auguste

1898 Mission Pavie: Indo-Chine. 1879-1895. Études diverses: II. Recherches sur l'histoire du Cambodge, du Laos et du Siam. Paris: Ernest Leuroux.

Phoumirath, Tongrith

2004 Kindong. Tradition, Memory, and Identity in Lao Weddings. Canberra: Australian Studies Graduate Program. (Doctoral Thesis, Australian National University)

\section{Pottier, Richard}

2004 Santé et société au Laos, 1973-1978. Le système de santé lao et ses possibilités de développement. Le cas de la zone de santé de Tha Düa. Paris: Comité de coopération avec le Laos.

\section{Stuart-Fox, Martin}

1996 Buddhist Kingdom, Marxist State. The Making of Modern Laos. Bangkok: White Lotus. (Studies in Southeast Asian History, 2)

2001 Historical Dictionary of Laos. London: The Scarecrow Press. (Asian/Oceanian Historical Dictionaries Series, 35) [2nd Ed.]

2005 Politics and Reform in the Lao People's Democratic Republic. Perth: Asia Research Center. (Working Paper, 126)

2006 The Political Culture of Corruption in the Lao PDR. Asian Studies Review 30/1: 59-75.

\section{Taillard, Christian}

1979 Le dualisme urbain-rural au Laos et la récupération de l'idéologie traditionnelle. ASEMI - Asie du Sud-Est et Monde Insulindien 10/1: 41-56.

1992 Les régimes politiques passent ... les échelles d'organisation de l'espace demeurent. Essai sur l'héritage des systèmes politiques thaï au Laos. In: J. Matras-Guin et C. Taillard (éds.), Habitations et habitats d'Asie du SudEst continentale. Pratiques et représentations de l'espace; pp. 305-342. Paris: Éditions L'Harmattan.

Testart, Alain

1997 Les trois modes de transfert. Gradhiva 21: 39-58.

2007 Critique du don. Études sur la circulation non marchande. Paris: Syllepse.

Weber, Max

1959 Le savant et le politique. Paris: UGE. (Collection 10/18) 
